\title{
CCL4 Levels Differ between Aspirin-Tolerant and Aspirin-Intolerant Patients with Asthma
}

\author{
Juan Raymundo Velazquez ${ }^{1}$, Gabriela Soid-Raggi' ${ }^{2}$ Luis Manuel Teran', \\ Gandhi Fernando Romero-Pavon1, Fernando Ramírez-Jimenez ${ }^{1}$ \\ ${ }^{1}$ Instituto Nacional de Enfermedades Respiratorias (INER), Mexico City, Mexico \\ ${ }^{2}$ Facultad de Ciencias, Universidad Nacional Autónoma de México (UNAM), Mexico City, Mexico \\ Email: velazquez_juan@outlook.com
}

How to cite this paper: Velazquez, J.R., Soid-Raggi, G., Teran, L.M., Romero-Pavon, G.F. and Ramírez-Jimenez, F. (2017) CCL4 Levels Differ between Aspirin-Tolerant and Aspirin-Intolerant Patients with Asthma. Health, 9, 1467-1474.

https://doi.org/10.4236/health.2017.911107

Received: July 14, 2017

Accepted: October 15, 2017

Published: October 18, 2017

Copyright $\odot 2017$ by authors and Scientific Research Publishing Inc. This work is licensed under the Creative Commons Attribution International License (CC BY 4.0).

http://creativecommons.org/licenses/by/4.0/

\begin{abstract}
Aspirin-exacerbated respiratory disease (AERD) is an asthma phenotype characterized by nasal polyps, chronic hypertrophic eosinophilic sinusitis, asthma, and sensitivity to aspirin. Unawareness of this disease by patients and their physicians may have serious consequences because of the risk of near-fatal asthma. Its confirmative diagnosis can be established only by provocative aspirin challenge, which represents a potential risk for patient health. Purpose: Because CCL4 plays an important role in several pulmonary conditions, we tested its immunoreactivity in nasal lavages and sera from control subjects, and from aspirin-sensitive and non-aspirin-sensitive patients. Method: Immunoreactivity of CCL4 was measured in serum and nasal lavages from 30 healthy controls (HC), 23 patients with AERD, and 20 patients with aspirin-tolerant asthma (ATA). Additionally, a serum biobank contained sera from $90 \mathrm{HC}, 83$ patients with AERD, and 69 patients with ATA was employed. Results: The serum immunoreactivity of CCL4 could distinguish both types of asthma phenotypes. Conclusion: CCL4 may play an unexpected role in thephysiopathology of AERD.
\end{abstract}

\section{Keywords}

AERD, CCL4, Asthma

\section{Introduction}

Aspirin-exacerbated respiratory disease (AERD) consists of a clinical tetrad of nasal polyps, chronic hypertrophic eosinophilic sinusitis, asthma, and sensitivity to any Non-steroidal anti-inflammatory drug (NSAID), one of which is aspirin [1]. AERD is also considered a late-onset eosinophilic asthma phenotype [2]. The aspirin-triggered anaphylactoid response is not Immunoglobin E (IgE)- 
mediated, but is related with alterations in the lipooxygenase and cyclooxygenase pathways [3]. Aspirin or other cross-reacting NSAIDs act by inhibiting COX-1, which produces a rapid decrease in the synthesis of its products including PGE2. In the airways, PGE2 is considered the most immunomodulatory, anti-fibrotic and bronchodilating prostanoid. The imbalance induced by aspirin and other NSAIDs in lipid mediator production may favor the lipoxygenase pathway and its related molecules, causing a flow of proinflammatory leukotrienes. Ingestion of aspirin by patients with AERD may result in acute bronchoconstriction, vascular permeability, and eosinophil migration, manifesting clinically as acute rhinitis and asthma. Yet important, the role of prostaglandins and the effect of aspirin on COX-dependent metabolism are not sufficient to explain the pathophysiology of AERD; sensitivity to ingested aspirin is a marker of disease, not the cause of the disease [4] [5].

It is common for patients with asthma, and even for their medical caregivers, to be unaware of the AERD condition of the former. Because its confirmative diagnosis implies provocative aspirin challenges, along with the potential risk for patient health and the need for its implemented in specialized clinics, the majority of patients with AERD are not properly diagnosed [6]. On considering AERD as an asthma phenotype, the quest for cytokines to be utilized as specific markers of the disease acquires interest. Some genes have been associated with AERD [4]: at the protein level, CCL5/RANTES and CCL7/MCP3 were found significantly elevated in nasal lavages from patients with AERD in comparison with patients with Aspirin-tolerant asthma (ATA) [7].

The role of MIP- $1 \beta /$ Chemokine (C-C motif) ligand 4 (CCL4) in AERD has not been explored. It is known that CCL4 is involved in the induction and modulation of several inflammatory responses through the recruitment of leukocytes to sites of infection [8]. CCL4 signals through CCR5 receptor, this receptor is expressed at high levels in Th1, and almost absent in Th2 cells. Levels of CCL4 are increased in alcoholic liver disease [9], glomerulonephritis, acute renal transplant rejection [10], human sepsis [11], and advanced atherosclerosis lesions [12]. There are no reports regarding the role of CCL4 in asthma disease but there are some observations of higher levels of this cytokine in several pulmonary conditions such as chronic bronchitis [13], pulmonary fibrosis [14], and cystic fibrosis [15].

Because the role of CCL4 in the induction and modulation of inflammatory response through the recruitment of leukocytes to infection sites in pulmonary conditions, we decided to compare its production in sera and nasal lavages from patients with AERD and ATA.

\section{Materials and Methods}

Seventy three individuals were distributed into the following three different groups: Healthy control (HC) subjects without asthma and nasal polyps ( $n=$ $30)$; patients with AERD $(n=23)$, and patients with ATA $(n=20)$. The sub- 
jects were enrolled at the National Institute of Respiratory Diseases (INER), Department de Inmunogenetics and Allergy, in Mexico City. All participants were studied when clinically stable, and patients with asthma suspended their treatments 7 days prior to study initiation. Characteristics of the study group are depicted in Table 1. The protocol was approved by the hospital's Ethics Committee.

The Skin prick test (SPT) was carried out by means of a kit of 40 common inhalant allergens (ALK-Abello, Wallingford, CT, USA) in each subject.

The diagnosis of asthma was established by conventional lung function tests as follows: spirometry with bronchodilator response and methacholine challenge, according to Global Initiative for Asthma (GINA) and American Thoracic Society (ATS) guidelines. Intolerance to aspirin was established by nasal challenge with Lysine-aspirin (L-ASA) (Aspisol'; Bayer AG, Leverkusen, Germany) according the guidelines established by the European Academy of Allergology and Clinical Immunology (EAACI)/Global Allergy and Asthma European Network (GA²LEN).

Challenge-free nasal lavage samples were collected by instilling sterile physiological saline solution into each nostril. Aliquots of $0.5 \mathrm{~mL}$ were filtered in Amicon Ultra- 0.5 (Millipore, Billerica, MA, USA). Concentrated nasal lavage samples were maintained at $-20^{\circ} \mathrm{C}$ until their use. Additionally, peripheral blood was collected from each individual before nasal lavage and used for eosinophil counting and total IgE determination.

CCL4 immunoreactivity was tested in unfrozen nasal lavages and serum samples employing Human Cytokine Chemokine Magnetic (HCYTOMAG) Bead Panel (Millipore); sensitivity was $3 \mathrm{pg} / \mathrm{mL}$.

The Mann-Whitney $U$ test was utilized for comparison among groups, and the Spearman test was used for correlations.

Table 1. Demographic characteristics.

\begin{tabular}{cccc}
\hline & EREA $(\mathrm{n}=23)$ & ATA $(\mathrm{n}=20)$ & HC $(\mathrm{n}=30)$ \\
\hline Age, yr & $49(37-55)$ & $37(24-51)$ & $21(19-22)$ \\
Female gender, No (\%) & $15(65.5)$ & $9(45)$ & $17(56.6)$ \\
Atopy (\%) & $11(47.8)$ & $8(40)$ & $8(26.6)$ \\
Blood eosinophils count, No/mm & $520(337-802)$ & $300(100-850)^{* *}$ & $116(61-224)^{*}$ \\
Total IgE, pg/ml & $267(224-662)$ & $414(231-573)^{* *}$ & $266(213-338)$ \\
FEV1 (\%) & $104(92-112)^{* * *}$ & $83(77-93)^{* *}$ & $95(92-102)$ \\
Fall in NTF induced by L-ASA (\%) & $50(45-59)^{* * *}$ & $10(10-10)$ & $5.1(5.0-5.0)^{*}$ \\
\hline
\end{tabular}

Healthy controls (HD), Aspirin-exacerbated respiratory disease (AERD), Aspirin tolerant asthma (ATA), nasal total flow (NTF). Values are given as median (p25-p75). ${ }^{*} p<0.001$ HC vs AERD; ${ }^{* *} p<0.02$ ATA vs $\mathrm{HC} ;{ }^{* *} p<0.003$ AERD vs ATA. 


\section{Results}

Clinical data showed that blood eosinophils in patients with AERD and ATA did not differ, but both were significant higher than in the HC group. Total IgE was significantly higher ( $439 \pm 191$ vs. $281 \pm 107 \mathrm{ng} / \mathrm{mL} ; p<0.02)$ in the ATA compared with the HC group. IgE did no differ in any other intergroup comparison. AERD condition was confirmed by the fall in total nasal flow induced by L-ASA.

CCL4 immunoreactivity in nasal lavages was barely significantly higher in the AERD when compared to HC group $(p=0.05)$. ATA vs HC and ATA vs AERD groups did not differ (Figure 1(a)).

CCL4 concentration in sera from the same set of individuals was significantly higher $(p=0.003)$ in the AERD than in the ATA group, and significantly higher in the HC than in the ATA group $(p=0.001)$. Consequently, there was no difference between the HC and AERD groups (Figure 1(b)).

There was no positive correlation between nasal lavage and serum CCL4 in any group. When groups were arranged into atopic and non-atopic subjects, no relationship or significant intra- or intergroup differences was found.

Differences in CCL4 sera from subjects in the AERD and ATA groups encourage us to seek this trend in a larger set of samples. Employing a collection of HC $(n=90)$, AERD $(n=83)$, and ATA $(n=69)$ serum samples, we tested the biomarker character of CCL4. CCL4 levels were significantly higher in both AERD and HC groups than in the ATA group ( $p<0.001$, for both). Once again, the $\mathrm{HC}$ and AERD groups did not differ (Figure 2).

\section{Discussion}

The use of risk-free methods based on cytokine immunoreactivity would be of enormous importance to clinical practice for distinguishing between AERD and ATA. The mediators involved in the arachidonic acid pathway comprise the

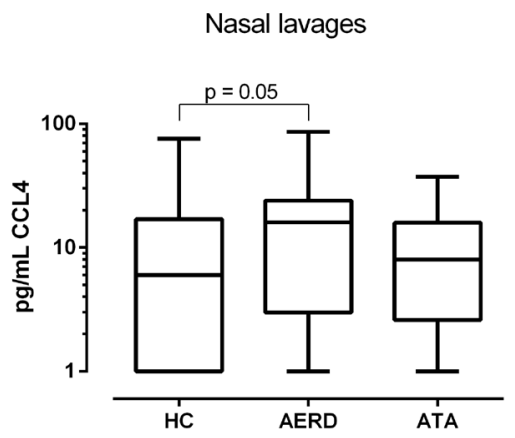

(a)

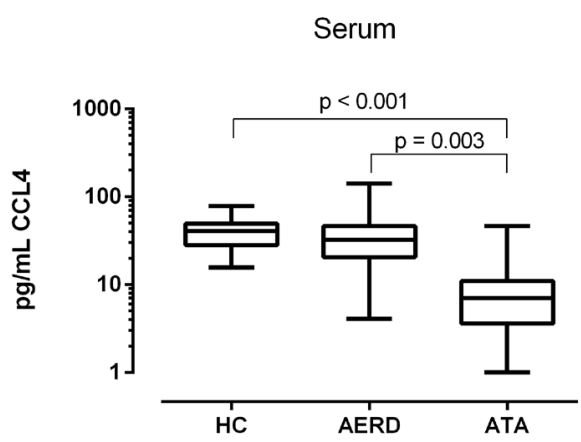

(b)

Figure 1. Cytokine immunoreactivity in nasal lavages (a) and sera (b) from Healthy control (HC) $(n=30)$; patients with Aspirin-exacerbated respiratory disease (AERD) $(n=$ $23)$, and patients with Aspirin-tolerant asthma (ATA) $(n=20)$. Significant differences after intergroup statistical analysis are represented. Cytokine (C-C motif) ligand 4 (CCL4) amounts are in $\mathrm{pg} / \mathrm{mL}$ in base- $10 \log$ scale for the $\mathrm{Y}$ axis. $P$ values are depicted above each comparison line. Whisker plots correspond to minimal-maximal values. 


\section{Sera collection}

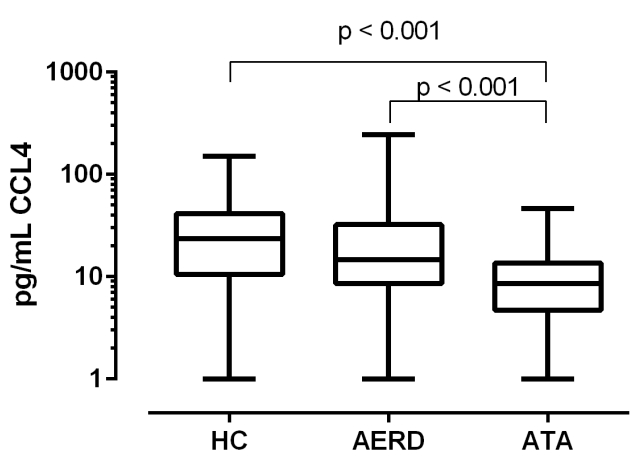

Figure 2. Cytokine immunoreactivity in a serum collection from Healthy controls HC ( $n$ $=90)$, patients with Aspirin-exacerbated respiratory disease (AERD) $(n=83)$, and patients with Aspirin-tolerant asthma (ATA) $(n=69)$. Significant differences in Chemokine (C-C motif) ligand 4 (CCL4) among the groups are represented. Amounts are in pg/mL in base-10 log scale for the $\mathrm{Y}$ axis. $P$ values are depicted above each comparison line. Whisker plots correspond to minimal-maximal values.

most plausible biomarkers for AERD. It is considered that the airway inflammatory response in allergic asthma is mediated by Th2 cytokines. Unfortunately, few of these have proven useful as AERD biomarkers [4].

CCL4 has an important role in the induction and modulation of several inflammatory responses through the recruitment of leukocytes to sites of infection [8]. CCL4 (such as CCL3 and CCL5) signals through CCR5; this receptor is expressed at high levels in Th1 and is nearly absent in Th2 cells [16]. CCL3 and CCL5 are important chemokines of airway hyperresponsiveness; in contrast, the role of CCL4 in asthma is uncertain. However, there are some reports of higher levels of this cytokine in several pulmonary conditions, such as chronic bronchitis [9], pulmonary fibrosis [14], and cystic fibrosis [15].

We found that CCL4 in nasal lavages was unable to distinguish between AERD and ATA. However, in serum, CCL4 was significantly higher in patients with AERD than in those with ATA. It is known that several cytokines in nasal lavages remain below the detection limit of commonly applied quantification assays [17]. Additionally, we found no correlation between nasal and systemic levels of CCL4. The lack of control over the dilution factor in nasal fluids would explain that finding. In our study, consistent results were found whether the studied group was smaller or larger. Indeed, the ATA group appeared to be a poor producer of systemic CCL4.

Diminished CCL4 production by CD4+ and CD8+ T cells in patients with allergic asthma has been reported by Grob et al. [18]. Despite that there was no correlation between cellular and serum levels of CCL4, the authors elucidated polarization away from the Th1 response, along with a Th2-response bias in patients with ATA.

According to our results, local production of CCL4 does not appear to be useful for distinguishing among asthma phenotypes. However, systemic CCL4 
levels in patients with AERD are in great proximity with those of healthy individuals, who usually present a more balanced Th1/Th2 cytokine production. This intriguing finding led us to hypothesize regarding a general mechanism that results in apparently higher CCL4 production in patients with AERD in comparison with those with ATA. In this context, an aspirin challenge that favors a Th2 response [19] would trigger the exacerbation of asthma symptoms in AERD patients. Whether CCL4 play a pivot role or is solely a product of a more complex and intriguing pathway in asthma phenotypes is a matter for further research. We are taking steps in that direction by analyzing nasal lavages and sera from patients with AERD and those with ATA using an aspirin time-course challenge.

\section{Conclusion}

Although asthma is considered a disorder of the respiratory system, it may possess a systemic component prone for use as specific biomarkers. The CCL4 role in asthma is not completely understood; its differential systemic production in patients with AERD and in those with ATA suggests an association with asthma phenotypes. Most likely, CCL4 and some of its regulator molecules are suitable for use as asthma phenotype biomarkers.

\section{Compliance with Ethical Standards}

\section{Funding}

This work was supported by Mexican National Council of Science and Technology (CONACyT-México) grant 160489.

\section{Conflict of Interest}

Author Soid-Raggi G declares that she has no conflict of interest. Author Terán LM declares that he has no conflict of interest, Romero-Pavón GF declares that he has no conflict of interest, Ramírez-Jiménez F declares that he has no conflict of interest and Velázquez JR declares that he has no conflict of interest.

\section{Ethical Approval}

All procedures performed in studies involving human participants were in accordance with the ethical standards of Instituto Nacional de Enfermedades Respiratorias committee and with the 1964 Helsinki declaration and its later amendments or comparable ethical standards.

\section{Informed Consent}

Informed consent was obtained from all individual participants included in the study.

\section{References}

[1] Lee, R.U. and Stevenson, D.D. (2011) Aspirin-Exacerbated Respiratory Disease: 
Evaluation and Management. Allergy, Asthma \& Immunology Research, 3, 3-10. https://doi.org/10.4168/aair.2011.3.1.3

[2] Wenzel, S.E. (2006) Asthma: Defining of the Persistent Adult Phenotypes. Lancet, 368, 804-813. https://doi.org/10.1016/S0140-6736(06)69290-8

[3] Babu, K.S. and Salvi, S.S. (2000) Aspirin and Asthma. Chest, 118, 1470-1476. https://doi.org/10.1378/chest.118.5.1470

[4] Velazquez, J.R. and Teran, L.M. (2013) Aspirin-Intolerant Asthma: A Comprehensive Review of Biomarkers and Pathophysiology. Clinical Reviews in Allergy \& Immunology, 45, 75-86. https://doi.org/10.1007/s12016-012-8340-0

[5] Walgama, E.S. and Hwang, P.H. (2017) Aspirin-Exacerbated Respiratory Disease. Otolaryngologic Clinics of North America, 50, 83-94. https://doi.org/10.1016/j.otc.2016.08.007

[6] Szczeklik, A. (1992) Aspirin-Induced Asthma: Pathogenesis and Clinical Presentation. Allergy Proceedings, 13, 163-173. https://doi.org/10.2500/108854192778817211

[7] Kupczyk, M., Kurmanowska, Z., Kuprys-Lipinska, I., Bochenska-Marciniak, M. and Kuna, P. (2010) Mediators of Inflammation in Nasal Lavage from Aspirin Intolerant Patients after Aspirin Challenge. Respiratory Medicine, 104, 1404-1409. https://doi.org/10.1016/j.rmed.2010.04.017

[8] Menten, P., Wuyts, A. and Van, D.J. (2002) Macrophage Inflammatory Protein-1. Cytokine \& Growth Factor Reviews, 13, 455-481. https://doi.org/10.1016/S1359-6101(02)00045-X

[9] Afford, S.C., Fisher, N.C., Neil, D.A., Fear, J., Brun, P., Hubscher, S.G., et al. (1998) Distinct Patterns of Chemokine Expression Are Associated with Leukocyte Recruitment in Alcoholic Hepatitis and Alcoholic Cirrhosis. The Journal of Pathology, 186, 82-89.

https://doi.org/10.1002/(SICI)1096-9896(199809)186:1<82::AID-PATH151>3.0.CO; $\underline{2-\mathrm{D}}$

[10] Adams, D.H., Hubscher, S., Fear, J., Johnston, J., Shaw, S. and Afford, S. (1996) Hepatic Expression of Macrophage Inflammatory Protein-1 Alpha and Macrophage Inflammatory Protein-1 Beta after Liver Transplantation. Transplantation, 61, 817-825. https://doi.org/10.1097/00007890-199603150-00024

[11] O’Grady, N.P., Tropea, M., Preas, H.L., Reda, D., Vandivier, R.W., Banks, S.M., et al. (1999) Detection of Macrophage Inflammatory Protein (MIP)-1 Alpha and MIP-1 Beta during Experimental Endotoxemia and Human Sepsis. The Journal of Infectious Diseases, 179, 136-141. https://doi.org/10.1086/314559

[12] Reape, T.J. and Groot, P.H. (1999) Chemokines and Atherosclerosis. Atherosclerosis, 147, 213-225.

[13] Capelli, A., Di, S.A., Gnemmi, I., Balbo, P., Cerutti, C.G., Balbi, B., et al. (1999) Increased MCP-1 and MIP-1 Beta in Bronchoalveolar Lavage Fluid of Chronic Bronchitics. The European Respiratory Journal, 14, 160-165. https://doi.org/10.1034/j.1399-3003.1999.14a27.x

[14] Emad, A. and Emad, V. (2007) Elevated Levels of MCP-1, MIP-Alpha and MIP-1 Beta in the Bronchoalveolar Lavage (BAL) Fluid of Patients with Mustard GasInduced Pulmonary Fibrosis. Toxicology, 240, 60-69.

[15] Brennan, S., Sly, P.D., Gangell, C.L., Sturges, N., Winfield, K., Wikstrom, M., et al. (2009) Alveolar Macrophages and CC Chemokines Are Increased in Children with Cystic Fibrosis. The European Respiratory Journal, 34, 655-661.

https://doi.org/10.1183/09031936.00178508 
[16] Odum, N., Bregenholt, S., Eriksen, K.W., Skov, S., Ryder, L.P., Bendtzen, K., et al. (1999) The CC-Chemokine Receptor 5 (CCR5) Is a Marker of, But Not Essential for the Development of Human Th1 Cells. Tissue Antigens, 54, 572-577.

https://doi.org/10.1034/j.1399-0039.1999.540606.x

[17] Boot, J.D., Chandoesing, P., de Kam, M.L., Mascelli, M.A., Das, A.M., Gerth van, W.R., et al. (2008) Applicability and Reproducibility of Biomarkers for the Evaluation of Anti-Inflammatory Therapy in Allergic Rhinitis. Journal of Investigational Allergology and Clinical Immunology, 18, 433-442.

[18] Grob, M., Schmid-Grendelmeier, P., Joller-Jemelka, H.I., Ludwig, E., Dubs, R.W., Grob, P.J., et al. (2003) Altered Intracellular Expression of the Chemokines MIP-1 Alpha, MIP-1 Beta and IL-8 by Peripheral Blood CD4+ and CD8+ T Cells in Mild Allergic Asthma. Allergy, 58, 239-245.

https://doi.org/10.1034/j.1398-9995.2003.00035.x

[19] Moon, H.G., Tae, Y.M., Kim, Y.S., Gyu, J.S., Oh, S.Y., Song, G.Y., et al. (2010) Conversion of Th17-Type into Th2-Type Inflammation by Acetyl Salicylic Acid via the Adenosine and Uric Acid Pathway in the Lung. Allergy, 65, 1093-1103.

https://doi.org/10.1111/j.1398-9995.2010.02352.x 